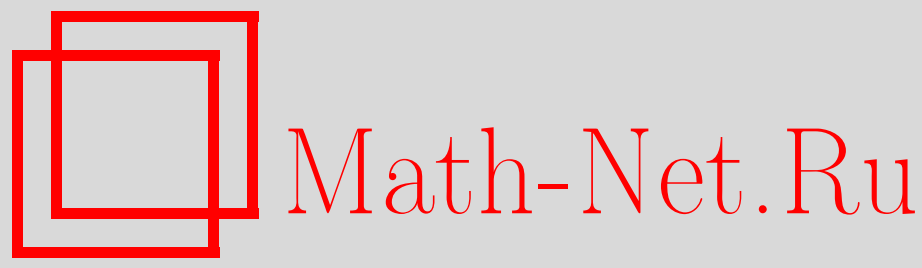

Й. Брюнинг, С. Ю. Доброхотов, Р. В. Некрасов, Расщепление нижних энергетических уровней в квантовой двойной яме в магнитном поле и туннелирование волновых пакетов в нанопроводах, ТМФ, 2013, том 175, номep 2, 206-225

DOI: https://doi.org/10.4213/tmf6882

Использование Общероссийского математического портала Math-Net.Ru подразумевает, что вы прочитали и согласны с пользовательским соглашением http: //www.mathnet.ru/rus/agreement

Параметры загрузки:

IP: 54.162 .27 .143

26 апреля 2023 г., $14: 18: 47$

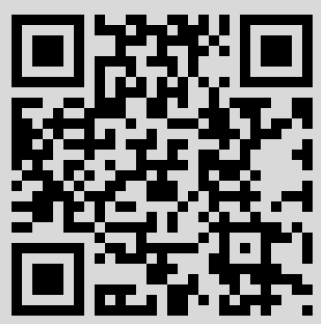


(C) 2013 г. Й. Брюнинг ${ }^{*}$ С. Ю. Доброхотов ${ }^{\dagger \ddagger}$, Р. В. Некрасов ${ }^{\dagger \dagger}$

\title{
РАСЩЕПЛЕНИЕ НИЖНИХ ЭНЕРГЕТИЧЕСКИХ УРОВНЕЙ В КВАНТОВОЙ ДВОЙНОЙ ЯМЕ В МАГНИТНОМ ПОЛЕ И ТУННЕЛИРОВАНИЕ ВОЛНОВЫХ ПАКЕТОВ В НАНОПРОВОДАХ
}

\begin{abstract}
Рассматривается задача о расщеплении нижних собственных значений двумерного оператора Шредингера с потенциалом типа двойной ямы при наличии однородного магнитного поля. Основной результат состоит в наблюдении о том, что с помощью частичного преобразования Фурье рассматриваемый оператор приводится к виду оператора Шредингера с (новым) потенциалом типа двойной ямы, но уже без магнитного поля. Благодаря этому наблюдению исследовано влияние магнитного поля на туннельные эффекты. Обсуждаются два способа вычисления расщепления нижних собственных значений: основанный на инстантоне и основанный на так называемой либрации. Полученный результат применяется для исследования туннелирования волновых пакетов в параллельных квантовых нанопроводах, помещенных в постоянное магнитное поле.
\end{abstract}

Ключевые слова: оператор Шредингера, потенциал типа двойной ямы, однородное магнитное поле, туннелирование, двойной квантовый провод, волновой пакет.

DOI: $10.4213 / \operatorname{tmf} 6882$

\section{1. ВВЕДЕНИЕ}

Мы рассматриваем задачу о расщеплении нижних собственных значений оператора Шредингера на плоскости с координатами $(y, z)$ с потенциалом типа двойной ямы

$$
V(y, z)=v_{1}(y)+\frac{\omega_{2}^{2} z^{2}}{2},
$$

где $v_{1}(y)$ - функция вида "одномерной двойной ямы", имеющей две невырожденные точки глобального минимума в точках $y= \pm a$ (например, $v_{1}=\omega_{1}^{2}\left(y^{2}-a^{2}\right)^{2} / 8 a^{2}$ ),

${ }^{*}$ Humboldt University, Berlin, Germany. E-mail: bruening@mathematik.hu-berlin.de

${ }^{\dagger}$ Институт проблем механики им. А. Ю. Ишлинского РАН, Москва, Россия. E-mail: dobr@ipmnet.ru

${ }^{\ddagger}$ Московский физико-технический институт, Москва, Россия.

E-mail: logno@rambler.ru 
и при наличии однородного магнитного поля, направленного перпендикулярно плоскости $(y, z)$. Соответствующий оператор Шредингера в калибровке Ландау имеет вид

$$
\widehat{H}=-\frac{h^{2}}{2} \frac{\partial^{2}}{\partial y^{2}}+\frac{1}{2}\left(-i h \frac{\partial}{\partial z}-b y\right)^{2}+V(y, z) .
$$

(Векторный потенциал $\mathbf{A}(x)=(0,0, b y)$.) Нас интересует асимптотика наименьших собственных значений этого оператора при $h \ll 1$. В отсутствие магнитного поля и в случае общего потенциала $V(y, z)$, не допускающего применения метода разделения переменных, эта задача (о квантовой частице в двойной потенциальной яме) довольно хорошо изучена и широко представлена в литературе (см., например, [1], [2]). Асимптотика нижних энергетических уровней имеет регулярный характер, и их степенная по параметру $h$ асимптотика легко получается с помощью осцилляторного приближения в окрестностях каждой из ям. Однако в силу симметрии между ямами такая степенная асимптотика с любой точностью по параметру $h$ имеет (по крайней мере) двукратное вырождение спектральных уровней. Учет туннельных эффектов снимает это вырождение и приводит к экспоненциально малому по $h$ расщеплению энергетических уровней, определяемому формулой $\Delta E=\mathcal{A}(h) e^{-\mathcal{J} / h}$.

Достаточно эффективные формулы для фазы $\mathcal{J}$, основанные на некоторых траекториях гамильтоновой системы с “туннельным" гамильтонианом $p^{2} / 2-V(x)$ (здесь и ниже в этом разделе $\left.p=\left(p_{y}, p_{z}\right), x=(y, z)\right)$, представлены во многих работах (см., например, [1]), эффективные асимптотические формулы для амплитуды $\mathcal{A}(h)$ приведены в работах [2], [3] (см. также [4]). При наличии магнитного поля поведение нижней части спектра оператора $\widehat{H}$ по-прежнему носит регулярный характер и для нахождения нижних энергетических состояний также может быть использовано осцилляторное приближение, асимптотические формулы также дают степенное вырождение соответствующих собственных значений. Они также отличаются друг от друга на экспоненциально малую величину, однако развитые в указанных выше работах методы вычисления величины их расщепления для ситуации с магнитным полем не работают. Причина состоит в следующем: для описания туннельных эффектов используются асимптотики метода Вентцеля-Крамерса-Бриллюэна с "чисто мнимыми фазами" $\varphi(x) e^{-S(x) / h}$, где $S(x)$ вещественнозначна и $S(x) \geqslant 0$. Подстановка такой функции в уравнение $\widehat{H} \psi=E \psi$ с последующим приравниванием коэффициентов при $h$ в нулевой степени приводит к уравнению Гамильтона-Якоби с “туннельным" гамильтонианом $-p^{2} / 2+V(x)$ (или, более удобным, $p^{2} / 2-V(x)$ ), отличающимся знаком при $p^{2}$ от "стандартного классического" гамильтониана $p^{2} / 2-V(x)$, но по-прежнему вещественнозначным. При наличии магнитного поля соответствующий гамильтониан вне зависимости от калибровки становится комплекснозначным и равным $(-i p-A(x))^{2} / 2+V(x)$, что и делает принципиально невозможным прямо использовать методы из цитированных выше статей.

Основным результатом этой работы является наблюдение (раздел 2) о том, что, по крайней мере в рассматриваемом примере, возможно сведение спектральной задачи для оператора $\widehat{H}$ к спектральной задаче для оператора Шредингера с потенциалом типа двойной потенциальной ямы, но уже без магнитного поля. Это наблюдение позволяет получить формулы для расщепления нижних энергетических уровней исходного магнитного оператора Шредингера. В разделе 3 обсуждаются алгоритмы 
расчета этого расщепления и оценивается влияние магнитного поля на расщепление. В качестве приложения в разделе 4 рассмотрен пример прохождения волнового пакета вдоль сдвоенного "квантового" провода в присутствии однородного магнитного поля. В плоскости, ортогональной такому сдвоенному проводу, потенциал удерживающих сил имеет вид двойной потенциальной ямы. Это приводит к туннелированию волновых пакетов между двумя проводами в процессе их прохождения. При помощи формулы для расщепления выведены формулы для времени перехода пакета из одного провода в другой с учетом наличия магнитного поля.

\section{2. ПЕРЕХОД ОТ КВАНТОВОЙ ДВОЙНОЙ ЯМЫ В МАГНИТНОМ ПОЛЕ К ДВОЙНОЙ ЯМЕ БЕЗ МАГНИТНОГО ПОЛЯ}

Сначала покажем, как в рассматриваемом случае можно “избавиться" от магнитного потенциала. Применим (частичное) преобразование Фурье по отношению к переменной $z$, определенное формулой

$$
\widetilde{\psi}\left(y, p_{z}\right)=\frac{1}{\sqrt{2 \pi h}} \int_{-\infty}^{\infty} \psi(y, z) e^{-i \omega_{2} p_{z} z / h} d z .
$$

Введем новые "смешанные" переменные: $x_{1}=y$ и $x_{2}=p_{z}$. Тогда в этом представлении оператор $\widehat{H}$ (см. формулу (2)) принимает вид

$$
\widehat{H}^{\prime}=\frac{\hat{p}_{1}^{2}}{2}+\frac{\hat{p}_{2}^{2}}{2}+v_{1}\left(x_{1}\right)+\frac{\left(\omega_{2} x_{2}-b x_{1}\right)^{2}}{2},
$$

где операторы $\hat{p}_{1}=-i h \partial / \partial x_{1}$ и $\hat{p}_{2}=-i h \partial / \partial x_{2}$ соответствуют прежним операторам $-i h \partial / \partial y$ и домножению на $-\omega_{2} z$. Сумма двух последних членов в (3) представляет собой функцию новых координат $\mathbf{x}^{\prime}$. Обозначим ее как

$$
\widetilde{V}=v_{1}\left(x_{1}\right)+\frac{\left(\omega_{2} x_{2}-b x_{1}\right)^{2}}{2} .
$$

Теперь магнитное поле входит в оператор $\widehat{H}^{\prime}$ лишь как параметр потенциала $\widetilde{V}$, который "сохраняет" свою инвариантность по отношению к инверсии $x_{1,2} \rightarrow-x_{1,2}$. Его минимумы расположены в точках $\left(x_{1}, x_{2}\right)=\left(a, b a / \omega_{2}\right)$ и $\left(x_{1}, x_{2}\right)=-\left(a, b a / \omega_{2}\right)$, где через $a>0$ обозначен минимум потенциала $v_{1}$. Таким образом, в рассматриваемом случае спектральная задача для оператора $\widehat{H}$ с магнитным полем сводится $к$ хорошо изученной задаче о собственных состояниях в двойной яме без магнитного поля.

\section{3. ФОРМУЛЫ ДЛЯ НИЖНИХ СОСТОЯНИЙ И РАСЩЕПЛЕНИЯ КВАЗИВЫРОЖДЕНИЯ}

Собственные функции оператора (3), соответствующие нижним энергетическим уровням, могут быть построены с экспоненциальной точностью, например, при помощи туннельного канонического оператора Маслова (см. [1]). В окрестности "дна ям" спектр оператора (3) имеет регулярный характер, асимптотика при $h \rightarrow 0$ соответствующих состояний может быть вычислена достаточно явно.

1. Нижние состояния имеют "асимптотическое вырождение" при $h \rightarrow 0$ : они могут быть разбиты на пары с экспоненциально тонким расщеплением энергетических 
уровней. Обозначим такую пару $\chi_{n}^{+}$и $\chi_{n}^{-}$, здесь состояния занумерованы квантовыми числами (двойным индексом): $n=\left(n_{1}, n_{2}\right), n_{i}=0,1,2, \ldots$. Соответствующие собственные значения обозначим как $\varepsilon_{n}^{ \pm}$.

2. Значения $\varepsilon_{n}^{ \pm}$с малыми номерами $n=\left(n_{1}, n_{2}\right)$ со степенной точностью могут быть найдены в осцилляторном приближении в окрестности минимума потенциала $\widetilde{V}$ (точнее сказать, в одном из двух его минимумов, но поскольку между ямами имеется симметрия, то уровни, соответствующие разным ямам, совпадают):

$$
\varepsilon_{n}^{ \pm}=h \omega_{1}^{\prime}\left(n_{1}+\frac{1}{2}\right)+h \omega_{2}^{\prime}\left(n_{2}+\frac{1}{2}\right)+o(h),
$$

где $\omega_{1}^{\prime}>0, \omega_{2}^{\prime}>0$ и

$$
\omega^{\prime 2}{ }_{1,2}^{2}=\frac{1}{2}\left(b^{2}+\omega_{1}^{2}+\omega_{2}^{2} \mp \sqrt{\left(b^{2}+\omega_{1}^{2}+\omega_{2}^{2}\right)^{2}-4 \omega_{1}^{2} \omega_{2}^{2}}\right),
$$

где введено обозначение $\omega_{1}=v_{1}^{\prime \prime}(a)$. Теория возмущений позволяет вычислить поправки к формуле (4), однако нам они не понадобятся. В дальнейшем мы ограничимся самыми менъшими энергетическими состояниями, т.е. случаем, когда $n_{1}=n_{2}=0$, и индекс $n=0$ будем опускать.

3. Со степенной точностью асимптотика собственных функций задается суперпозицией функций $\chi_{\text {left }}$ и $\chi_{\text {right }}$, соответствующих осцилляционному приближению в первой и во второй ямах потенциала $\widetilde{V}$ :

$$
\begin{array}{rlrl}
\chi_{0}^{+} & =\chi_{\text {left }}+\chi_{\text {right }}+O(h), & \chi_{0}^{-} & =\chi_{\text {left }}-\chi_{\text {right }}+O(h), \\
\chi_{\text {left }} & =C e^{-\left(\omega_{1}^{\prime} y_{-}^{2}+\omega_{2}^{\prime} z_{-}^{2}\right) / 2 h}, & \chi_{\text {right }}=C e^{-\left(\omega_{1}^{\prime} y_{+}^{2}+\omega_{2}^{\prime} z_{+}^{2}\right) / 2 h},
\end{array}
$$

где $y_{ \pm}, z_{ \pm}$- ортогональные координаты в окрестности точки $\left(a, b a / \omega_{2}\right)$ и, соответственно, точки $\left(-a,-b a / \omega_{2}\right)$, в которых квадратичные формы потенциала $\widetilde{V}$ диагональны:

$$
V=\frac{\omega_{1}^{\prime 2}}{2} y_{ \pm}^{2}+\frac{\omega_{2}^{\prime 2}}{2} z_{ \pm}^{2}+O\left(\left(y_{ \pm}^{2}+z_{ \pm}^{2}\right)^{3 / 2}\right),
$$

а $C=1 / \sqrt{\pi \omega_{1} \omega_{2} h}$ - нормировочный коэффициент. Эти координаты могут быть вычислены по следующим формулам:

$y_{ \pm}=\left(x_{1} \mp a\right) \cos \phi+\left(x_{2} \mp a b / \omega_{2}\right) \sin \phi, \quad z_{ \pm}=-\left(x_{1} \mp a\right) \sin \phi+\left(x_{2} \mp a b / \omega_{2}\right) \cos \phi$, где

$$
\operatorname{tg} \phi=-\frac{2 b \omega_{2}}{b^{2}+\omega_{1}^{2}-\omega_{2}^{2}-\sqrt{\left(b^{2}+\omega_{1}^{2}+\omega_{2}^{2}\right)^{2}-4 \omega_{1}^{2} \omega_{2}^{2}}} .
$$

Собственные значения $\varepsilon^{ \pm}$, соответствующие функциям (6), имеют одинаковые степенные разложения по параметру $h$ в любом порядке теории возмущений, они отличаются на экспоненциально малое выражение:

$$
\varepsilon^{-}-\varepsilon^{+}=\mathcal{A}(h) e^{-\mathcal{J}(h) / h}(1+O(h)) .
$$

Асимптотическое представление для (7) не единственно, имеются, по крайней мере, два способа и две формулы для вычисления $\mathcal{J}(h)$ и $\mathcal{A}(h)$. Для полноты изложения опишем оба способа. В основе первого способа (назовем его способ $\mathbf{A}$ )

3 Теоретическая и математическая физика, т. 175, № 2, 2013 г. 


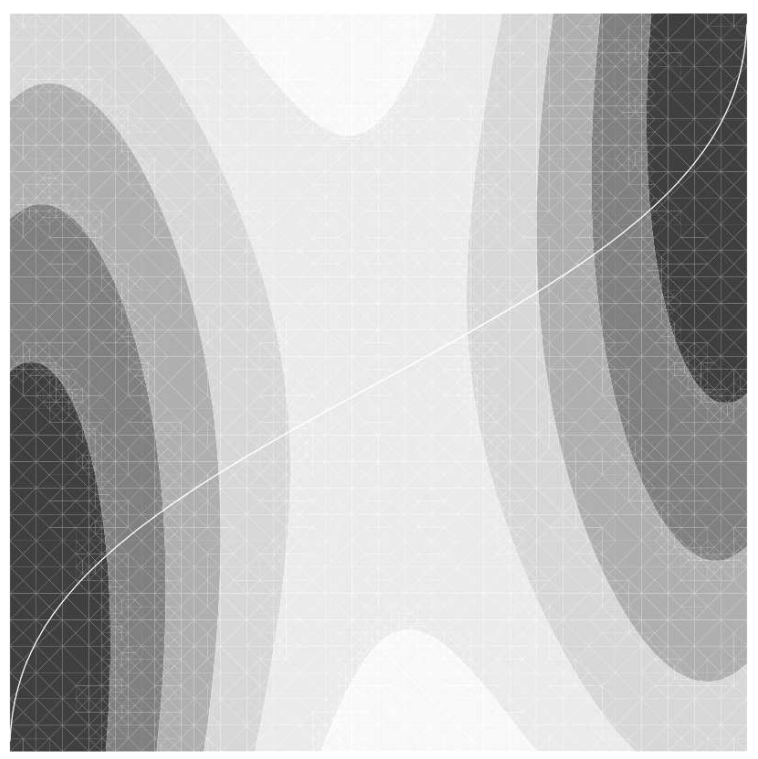

Рис. 1. Инстантон на фоне линий уровня потенциала $\widetilde{V}\left(x_{1}, x_{2}\right)$.

лежит инстантон-сингулярная траектория, соединяющая две вершины перевернутого потенциала; в основании второго способа (назовем его способ Б) лежит либрация - периодическая траектория гамильтоновой системы с перевернутым потенциалом с некоторой энергией $\widetilde{E}$.

Фаза $\mathcal{J}(h)$ в первой формуле вычислена во многих работах (в том числе в [1]), достаточно явные формулы для амплитуды $\mathcal{A}(h)$, основанные на траекториях линейной гамильтоновой системы (системы в вариациях), получены в работах [2]; формулы для $\mathcal{J}(h), \mathcal{A}(h)$ во втором случае (способ Б) выведены в работе [3] (см. также [4]).

Способ А. Вычисление расщепления двух самых нижних уровней по "инстантонной" формуле разбивается на следующие шаги.

Шаг 1. Расчет инстантонного действия. Показатель экспоненты $\mathcal{J}(h)$ в данном случае не зависит от $h$, и мы будем обозначать его через $S$. Величина $S$ вычисляется вдоль траектории $X(t)=\left(x_{1}(t), x_{2}(t)\right)$, соединяющей (за бесконечное время) точки неустойчивого равновесия в ньютоновой системе с перевернутым потенциалом $-\widetilde{V}\left(x_{1}, x_{2}\right)$. Именно,

$$
S=\int_{-\infty}^{+\infty}\left(\frac{|\dot{X}(t)|^{2}}{2}+\widetilde{V}(X(t))\right) d t
$$

есть действие вдоль траектории $X(t)=\left(x_{1}(t), x_{2}(t)\right)$, соединяющей точки максимума перевернутого потенциала $\widetilde{V}$ и являющейся решением уравнения Ньютона

$$
\begin{aligned}
& \ddot{x}_{1}=\frac{\partial \widetilde{V}}{\partial x_{1}}=v_{1}^{\prime}\left(x_{1}\right)+\omega_{2}\left(\omega_{2} x_{2}-b x_{1}\right), \\
& \ddot{x}_{2}=\frac{\partial \widetilde{V}}{\partial x_{2}}=-b\left(\omega_{2} x_{2}-b x_{1}\right) .
\end{aligned}
$$




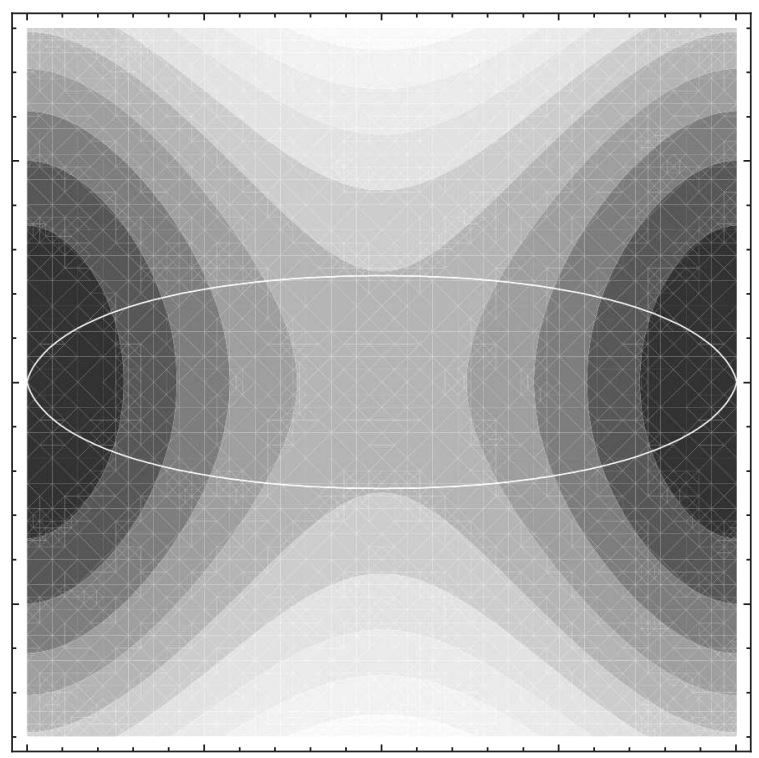

Рис. 2. Оба инстантона в исходных координатах на фоне линий уровня потенциала $V(y, z)$.

Такая траектория известна как инстантон (см. рис. 1). В фазовом пространстве $\left(x_{1}, x_{2}, p_{1}=\dot{x}_{1}, p_{2}=\dot{x}_{2}\right)$ такая траектория имеет в качестве прообраза на плоскости $\left(x_{1}, x_{2}\right)$ две компоненты, отличающиеся направлением движения (от первого дна ко второму и наоборот). Проекция этой траектории на плоскость $\left(x_{1}, p_{2}\right)$ позволяет рассмотреть инстантон в исходных координатах $\left(y=x_{1}, z=p_{2} / \omega_{2}\right)$ (см. рис. 2). Система (9) в таких координатах имеет вид

$$
\ddot{y}=v_{1}^{\prime}(y)-b \dot{z}, \quad \ddot{z}=\omega_{2}^{2} z-b \dot{y} .
$$

Действие (8) можно вычислить с помощью принципа наименьшего действия Гамильтона, минимизируя функционал (8) на непрерывных кривых, соединяющих точки $\left(a, a b / \omega_{2}\right)$ и $-\left(a, a b / \omega_{2}\right)$ (вершины потенциала $\left.-\tilde{V}\right)$. Учитывая равный вклад в $(8)$ от симметричных участков инстантона до и после прохождения седловой точки $(0,0)$, мы используем только одну половину, затем делаем замену времени $t$ на новое время $\tau=e^{-t}$ (чтобы избавиться от неограниченности времени $t$ ). Запишем это:

$$
S=2 \int_{0}^{+\infty}\left(\frac{|\dot{X}(t)|^{2}}{2}+\widetilde{V}(X(t))\right) d t=2 \int_{0}^{1}\left(\frac{|\dot{X}(\tau)|^{2}}{2} \tau+\frac{\tilde{V}(X(\tau))}{\tau}\right) d \tau .
$$

Численный расчет минимумов этого функционала для разных значений величины магнитного поля показал следующую зависимость от магнитного поля: действие $S$ возрастает при увеличении магнитного поля (рис. 3). Этот результат можно объяснить следующим образом. С ростом магнитного поля увеличивается расстояние между концами инстантона $x^{\prime}=\left(a, b a / \omega_{2}\right)$ и $-x^{\prime}=\left(-a,-b a / \omega_{2}\right)$, в то время как его максимальный и минимальный (а следовательно, и характерный) импульсы остаются неизменными: $p_{\min }=0, p_{\max }=\sqrt{2 \widetilde{V}(0,0)}$. С другой стороны, действие $S$ (действие Мопертюи) представляется как $\int_{-x^{\prime}}^{x^{\prime}} p d x \approx\left|x^{\prime}\right| p_{\max }$. 


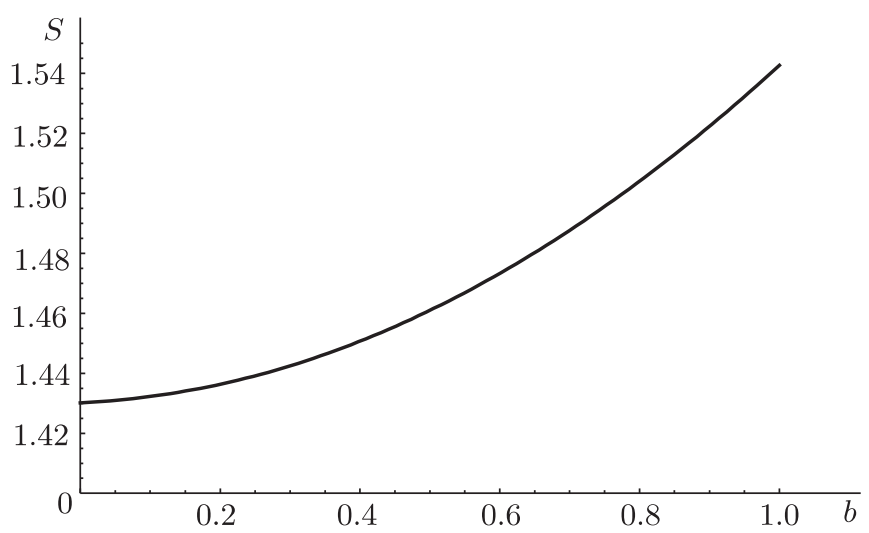

Рис. 3. Зависимость инстантонного действия как функция величины магнитного поля при фиксированных остальных параметрах $\left(a=\omega_{1}=\omega_{2}=1\right)$.

Шаг 2. Вычисление предэкспоненциального множителя (амплитуды расщепления)

$$
\mathcal{A}=\frac{4}{\sqrt{\pi}} \sqrt{\omega_{1}^{\prime} \omega_{2}^{\prime} h}|\dot{X}(0)| \frac{1}{(C n, n)} \frac{1}{J}
$$

разбивается на вычисление следующих сомножителей.

Шаг 2а. Множитель $\sqrt{\omega_{1}^{\prime} \omega_{2}^{\prime} h}$. Прямые вычисления показывают, что $\omega_{1}^{\prime} \omega_{2}^{\prime}=$ $\omega_{1} \omega_{2}$ (см. формулу (5)). Поэтому множитель $\sqrt{\omega_{1}^{\prime} \omega_{2}^{\prime} h}$ не зависит от магнитного поля, а определяется только характеристиками исходного потенциала $V$ и малостью параметра $h$.

Шаг 2б. Множитель $|\dot{X}(0)|$ равен скорости инстантона в седловой точке потенциала $-\widetilde{V}\left(x_{1}, x_{2}\right)$, совпадающей с серединой инстантона. Из закона сохранения энергии, написанного для середины и конца инстантона, имеем $|\dot{X}(0)|^{2} / 2-\widetilde{V}(0,0)=0$. Поэтому множитель $|\dot{X}(0)|=\sqrt{2 v_{1}(0)}$ также не зависит от магнитного поля, а определяется только характеристиками исходного потенциала $V$ (для модельного потенциала вида $v_{1}=\omega_{1}^{2}\left(y^{2}-a^{2}\right)^{2} / 8 a^{2}$ это $\left.|\dot{X}(0)|=\omega_{1} a / 2\right)$.

Шаг 2в. Множитель $J^{-1}=\lim _{t \rightarrow \infty} e^{\left(\omega_{1}^{\prime}+\omega_{2}^{\prime}\right) t} \operatorname{det} Z(t)$ определяется через решение системы в вариациях к траектории $X(t) .(2 \times 2)$-матрица $Z(t)$ удовлетворяет следующей задаче:

$$
\ddot{Z}_{i j}(t)=\left.\sum_{k=1}^{2} \frac{\partial^{2} \widetilde{V}}{\partial x_{i} \partial x_{k}}\right|_{x=X(t)} Z_{k j}(t), \quad Z(0)=E, \quad Z(\infty)=0,
$$

где $E$ - единичная $(2 \times 2)$-матрица.

Чтобы вычислить этот фактор, сначала нужно найти решение уравнения (10). Оно может быть найдено при помощи принципа наименьшего действия. В этом случае мы должны использовать его обобщение на случай матричнозначной неизвестной функции

$$
Z(\tau)=\operatorname{Arg} \min _{Z(\tau) \in C([0,1])^{4}} \operatorname{Tr} \int_{0}^{1} \tau \frac{\dot{Z} \dot{Z}^{\mathrm{T}}}{2}+\frac{Z \widetilde{V}(\mathcal{X}(\tau)) Z^{\mathrm{T}}}{\tau} d \tau, \quad Z(0)=0, \quad Z(1)=E .
$$




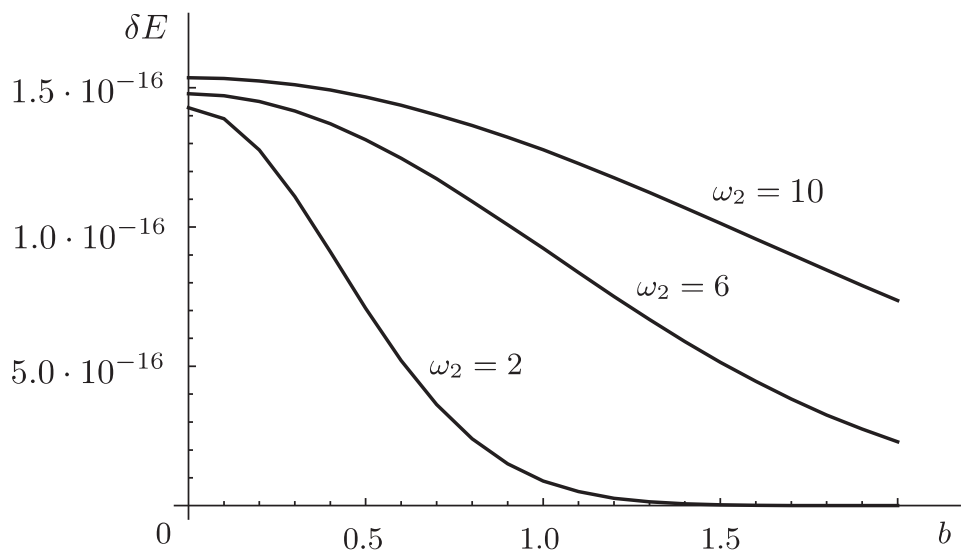

Рис. 4. Расщепление $\delta E=\varepsilon^{-}-\varepsilon^{+}$как функция параметра $b$ при разных значениях параметра $\omega_{2}$ при $\omega_{1}=6, a=1, h=0.1$.

После нахождения минимума этого функционала мы находим предел

$$
J^{-1}=\lim _{t \rightarrow \infty} e^{\left(\omega_{1}^{\prime}+\omega_{2}^{\prime}\right) t} \operatorname{det} Z(t)
$$

Шаг 2г. Множитель $(C n, n)$ получается из единичного вектора $n$, нормального к $|\dot{X}(0)|$, и матрицы $C=\dot{Z}(0)$, где $Z(t)$ - решения системы уравнений в вариациях (см. шаг 2в). Для вычислений этой матрицы, однако, было удобнее воспользоваться соотношением

$$
C=\left.\frac{\partial p}{\partial x}\right|_{p=\dot{\mathcal{X}}(0)}=\frac{\partial^{2} S_{1}}{\partial x^{2}},
$$

где функция $S_{1}$ удовлетворяет следующему уравнению:

$$
\begin{aligned}
S_{1}\left(x_{1}, x_{2}\right) & =\min _{\mathcal{X}(\tau) \in C([0,1])} \int_{0}^{1} \tau \frac{|\dot{\mathcal{X}}(\tau)|^{2}}{2}+\frac{\tilde{V}(\mathcal{X}(\tau))}{\tau} d \tau, \\
\mathcal{X}(0) & =\left(a, \frac{a b}{\omega_{2}}\right), \quad \mathcal{X}(1)=\left(x_{1}, x_{2}\right) .
\end{aligned}
$$

Поэтому соответствующий множитель может быть вычислен методом, подобным используемому при расчете на шаге 1 . Соответственно, $(2 \times 2)$-матрица $C=\partial^{2} S_{1} / \partial x^{2}$ $=\partial p /\left.\partial x\right|_{p=\dot{\mathcal{X}}(0)}$.

Результаты расчета по этим формулам $S$ и $\mathcal{A}$ как функций параметров $b$ и $\omega_{2}$ таковы. Составляющая магнитного поля $b$ увеличивает действие инстантона $S$ и вместе с ним уменьшает величину расщепления нижних уровней. Параметр $\omega_{2}$ (как будет показано ниже, для задачи с проводами этот параметр также соответствует величине магнитного поля в одном из поперечных направлений), наоборот, уменьшает величину действия, и при достаточно высоких его значениях действие выходит на определенную константу и тем самым перестает влиять на расщепление, а в игру вступает предэкспоненциальный множитель $A$. В свою очередь, параметр $A$ растет с ростом $\omega_{2}$. Таким образом, общий вывод следующий: с ростом параметра $b$ 


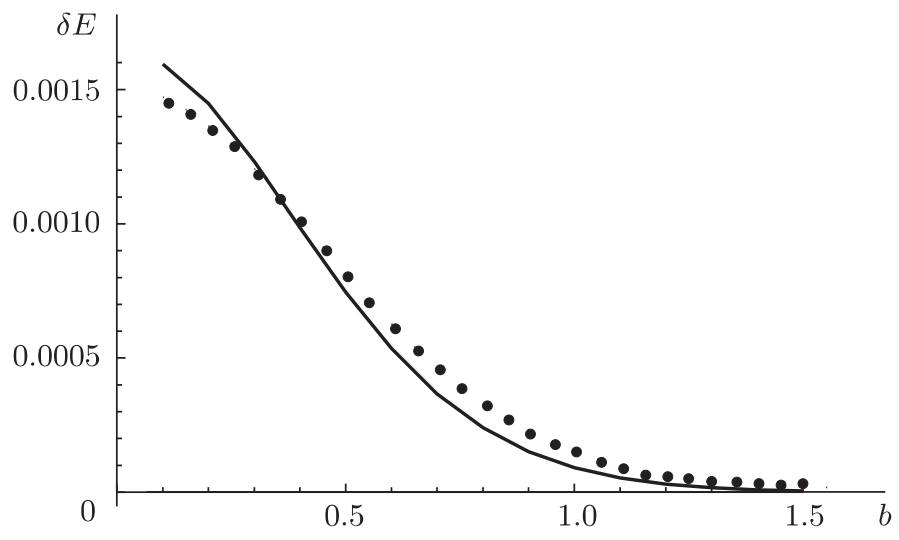

Рис. 5. Расщепление $\delta E=\varepsilon^{-}-\varepsilon^{+}$как функция параметра $b$ при $\omega_{2}=\omega_{1}=1$, $a=1, h=0.1$. Точки получены численно при помощи компьютерной программы Comsol, кривая соответствует расчету по инстантонной формуле (7).

(магнитного поля) расщепление уменьшается, а с ростом параметра $\omega_{2}$ расщепление увеличивается. Этот вывод иллюстрируют кривые на рис. 4. На рис. 5 изображены результаты расщепления, полученные В. Залипаевым с помощью численного анализа соответствующего уравнения Шредингера.

Способ Б. Вывод формул для фазы $\mathcal{J}$ и амплитуды $\mathcal{A}$ основан на следующем соображении (см. [3], а также работы [4]). Геометрические объекты, которые определяют асимптотические волновые функции в осцилляторном приближении, - это "маленькие" торы, имеющие в координатах $y_{ \pm}, z_{ \pm}$и соответствующих импульсных координатах $p_{y \pm}, p_{z \pm}$ следующий вид:

$$
\begin{array}{r}
\Lambda_{ \pm}=\left\{p_{y \pm}=-\sqrt{h \omega_{1}^{\prime}} \sin \phi_{1}, p_{z \pm}=-\sqrt{h \omega_{2}^{\prime}} \sin \phi_{2},\right. \\
\left.y_{ \pm}=\sqrt{h / \omega_{1}^{\prime}} \cos \phi_{1}, z_{ \pm}=\sqrt{h / \omega_{2}^{\prime}} \cos \phi_{2}\right\} .
\end{array}
$$

Они "почти инвариантны" относительно фазового потока, порожденного исходным гамильтонианом $H=p^{2} / 2+V(x)$, и лежат в окрестности минимума $V$ на уровне энергии $(h / 2)\left(\omega_{1}^{\prime}+\omega_{2}^{\prime}\right)$. Если заменить $V$ потенциалами гармонического осциллятора, то $\Lambda_{ \pm}-$торы Лиувилля, соответствующие квадратичным гамильтонианам. Движение по ним (приближенное) в силу гамильтоновой системы с гамильтонианом $H$ почти-периодично. Если соответствующий фазовый поток обозначить $g_{H}^{t}$, то мы получим

$$
\begin{array}{r}
g_{H}^{t} \Lambda_{ \pm}=\left\{p_{y \pm}=-\sqrt{h \omega_{1}^{\prime}} \sin \left(\phi_{1}+\omega_{1}^{\prime} t\right), p_{z \pm}=-\sqrt{h \omega_{2}^{\prime}} \sin \left(\phi_{2}+\omega_{2}^{\prime} t\right)\right. \\
\left.y_{ \pm}=\sqrt{h / \omega_{1}^{\prime}} \cos \left(\phi_{1}+\omega_{1}^{\prime} t\right), z_{ \pm}=\sqrt{h / \omega_{2}^{\prime}} \cos \left(\phi_{2}+\omega_{2}^{\prime} t\right)\right\}
\end{array}
$$

Инстантон - это траектория, соединяющая максимумы перевернутого потенциала, лежащие на предельном классическом нулевом уровне энергии, в то время как квантовый (“перевернутый”) уровень равен $-\mathcal{E}_{0}=-(h / 2)\left(\omega_{1}^{\prime}+\omega_{2}^{\prime}\right)+O\left(h^{2}\right)$. Поэтому 
более разумный с физической точки зрения объект, который определяет туннелирование, - это путь $\Gamma_{+}$, соединяющий в комплексном фазовом пространстве $\Lambda_{+}$и $\Lambda_{-}$, или путь $\Gamma_{-}$, определяющий движение в обратном направлении, и $\mathcal{J}=\pi J(\widetilde{E})$, где

$$
J(\widetilde{E})=\frac{1}{2 \pi} \oint \mathbf{p} d \mathbf{x}
$$

- действие пути (цикла) $\Gamma=\Gamma_{+}+\Gamma_{-}$. Соответствующая либрация и дает этот цикл (одну из его возможных реализаций), однако ее определение связано не с уровнем энергии $\mathcal{E}_{0}$, а с другим уровнем $\widetilde{E}$, поскольку "часть энергии" при туннелировании "расходуется" на движения, трансверсальные к этому пути. Замена инстантонного действия в формуле (7) на либрационное действие приводит к пересчету амплитуды, которая принимает простой вид: $\mathcal{A}=\omega_{1}^{\prime} h / \sqrt{\pi e}$, и формулу для расщепления через либрацию можно переписать в виде [1], [4]

$$
\varepsilon_{0}^{-}-\varepsilon_{0}^{+}=\frac{\omega_{1}^{\prime} h}{\sqrt{\pi e}} e^{-\pi J(\widetilde{E}) / h}(1+O(h)) .
$$

Заметим, что теперь $J(\widetilde{E}) / h$ зависит от $h$ (через $\widetilde{E}$ ). Алгоритм вычисления $J(\widetilde{E})$ следующий. Рассмотрим гамильтонову систему (с перевернутым потенциалом $-\widetilde{V}$ )

$$
\begin{array}{ll}
\dot{p}_{1}=\frac{\partial \widetilde{V}}{\partial x_{1}}=v_{1}^{\prime}\left(x_{1}\right)+\omega_{2}\left(\omega_{2} x_{2}-b x_{1}\right), & \dot{p}_{2}=\frac{\partial \widetilde{V}}{\partial x_{2}}=-b\left(\omega_{2} x_{2}-b x_{1}\right), \\
\dot{x}_{1}=p_{1}, & \dot{x}_{2}=p_{2} .
\end{array}
$$

Траектория, соединяющая вершины потенциала (инстантон), лежит на нулевом и "не квантовом" уровне энергии. Классическая траектория, отвечающая квантовой задаче, лежит на более низком уровне, отличном от $E=0$. Эта периодическая траектория в фазовом пространстве называется либрацией. Существование по крайней мере одной такой траектории для каждого уровня энергии установлено в работах [5]. Предположим, что в нашем случае для каждого уровня имеется только одна такая траектория. Обозначим ее период через $T=T(E)$. Изменяя $E$, мы получаем семейство либраций (периодических траекторий), гладко зависящих от параметра $E$ и в пределе $E \rightarrow 0$ переходящих в инстантонное решение. Вместо параметра $E$ мы можем также использовать переменную действия

$$
J=J(E)=\frac{1}{2 \pi} \oint \mathbf{p} d \mathbf{x},
$$

вычисленную вдоль этой траектории, и параметризовать этой переменной либрации, их периоды, энергетические уровни и т.д. Обозначим предельное (при $E \rightarrow 0$ ) действие через $J^{0}=J(0)$ и будем считать, что $J \in\left[J^{0}-\Delta, J^{0}\right)$, где $\Delta-$ малое положительное число, не зависящее от $h$. Таким образом, имеем либрацию в виде

$$
L(J)=\{p=\mathcal{P}(\Omega(J) t, J), x=\mathcal{X}(\Omega(J) t, J)\},
$$

где $\Omega(J)=2 \pi / T(J)$ - частота либрации, $\left.H\right|_{L}=E(J), J \in\left[J^{0}-\Delta, J^{0}\right)$. Функции $\mathcal{P}(\varphi, J), \mathcal{X}(\varphi, J)$ являются гладкими в заданном полуинтервале и $2 \pi$-периодичными по параметру $\varphi$. 
Движение в силу системы (13) в окрестности $L(J)$ в фазовом пространстве определяется соответствующей системой в вариациях. Базис ее решений можно составить из следующих функций. Два решения суть $(\dot{\mathcal{P}}, \dot{\mathcal{X}}),(\partial \mathcal{P} / \partial J+(\partial \Omega / \partial J)(\partial \mathcal{P} / \partial \Omega)$, $\partial \mathcal{X} / \partial J+(\partial \Omega / \partial J)(\partial \mathcal{X} / \partial \Omega))$. Два других, согласно теории Флоке-Ляпунова, представляются в виде решений Флоке:

$$
W(t)=\mathcal{W}(\Omega(J) t, J) e^{ \pm \beta(J) t}, \quad Z(t)=\mathcal{Z}(\Omega(J) t, J) e^{ \pm \beta(J) t},
$$

где при $J \in\left[J^{0}-\Delta, J^{0}\right) \mathcal{W}(\varphi, J)$ функции $\mathcal{Z}(\varphi, J)$ - гладкие $2 \pi$-периодичные по угловому аргументу $\varphi$ вектор-функции, $\beta(J)>0$ - показатель Флоке.

Заметим, что либрация - неустойчивая замкнутая траектория, и показатели Флоке в данном случае принимают чисто вещественные значения. Уравнение (приближенное) для $J(\widetilde{E})$ имеет вид

$$
E(J(\widetilde{E}))-\frac{1}{2} \beta(J(\widetilde{E})) h=-\frac{h}{2}\left(\omega_{1}^{\prime}+\omega_{2}^{\prime}\right) .
$$

Для практических вычислений удобно сначала найти энергию $\widetilde{E}=E(J(\widetilde{E}))$, переписав предыдущее уравнение в виде, пригодном для метода итераций:

$$
\widetilde{E}=\frac{1}{2} \tilde{\beta}(\widetilde{E}) h-\frac{h}{2}\left(\omega_{1}^{\prime}+\omega_{2}^{\prime}\right) .
$$

Таким образом, алгоритм вычисления $J(\widetilde{E})$ состоит из следующих шагов.

Шаг 1. Построим нулевое приближение $E^{0}$ к решению уравнения (17), отбросив в его правой части $(1 / 2) \tilde{\beta}(\widetilde{E}) h$ или $(1 / 2) \tilde{\beta}(\widetilde{E}) h-(h / 2) \omega_{2}^{\prime}$. Построим либрацию (замкнутую траекторию), соответствующую этой энергии. Возможность использования здесь принципа наименьшего действия в форме Гамильтона для поиска этой траектории не очевидна, поскольку неизвестно ее конечное положение в пространстве и времени (априори нам не известен ее период). Из соображений симметрии следует, что она проходит через начало координат. Поэтому мы подыскиваем начальные условия $\dot{x}_{1}(0), \dot{x}_{2}(0)$ в точке

$$
x_{1}(0)=0, \quad x_{2}(0)=0, \quad \frac{1}{2}\left(\dot{x}_{1}(0)\right)^{2}+\frac{1}{2}\left(\dot{x}_{2}(0)\right)^{2}=-E^{0},
$$

"пристрелкой", исходя из требования полного возвращения траектории в исходное положение. Этот шаг оказывается достаточно трудоемким в силу неустойчивости траектории и неизвестности ее периода.

Шаг 2. Далее строится последовательность $E^{1}, E^{2}, \ldots$ (и соответствующие либрации), достаточно быстро сходящаяся к $\widetilde{E}$ :

$$
E^{0}=-\frac{1}{2} \omega_{1}^{\prime} h-\frac{1}{2} \omega_{2}^{\prime} h, \quad E^{n+1}=E^{n}+\frac{1}{2} \tilde{\beta}\left(E_{n}\right) h .
$$

Реализовать этот шаг нетрудно, если реализован первый шаг.

Шаг 3. После этого по формуле (14) легко вычисляется $J(\widetilde{E})$. 


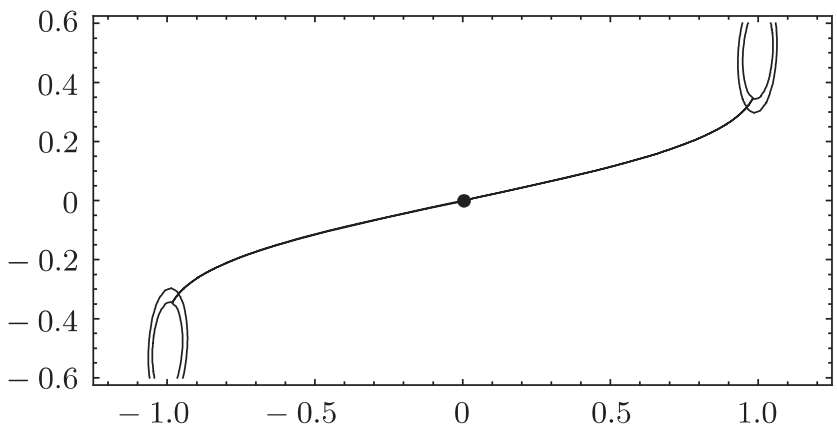

Рис. 6. Либрация в координатах $\left(x_{1}, x_{2}\right)$ и два уровня: уровень энергии $E=E_{0}$ (снаружи) и уровень, соответствующий энергии либрации, $\widetilde{E}=E_{0}+$ $\beta(\widetilde{E}) h / 2$ (внутри).

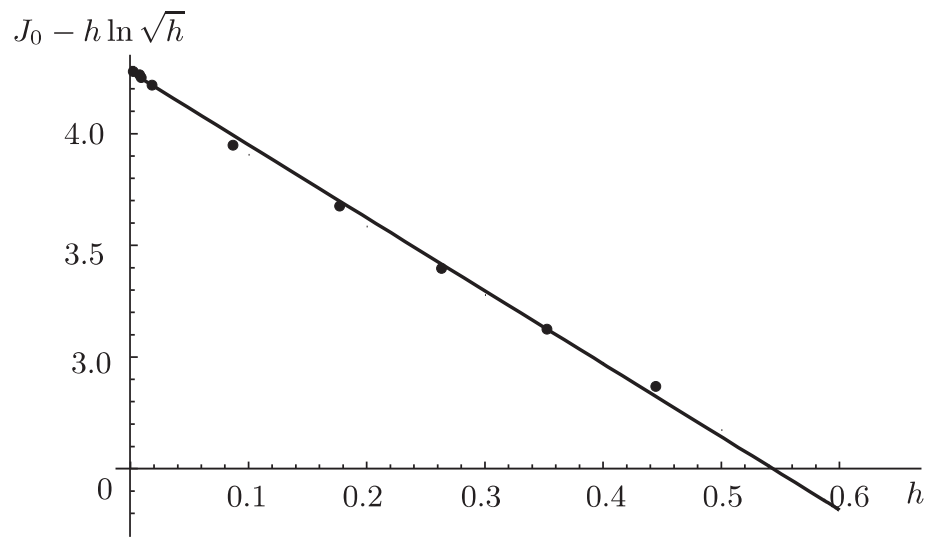

Рис. 7. График функций $J_{0}-h \ln \sqrt{h}$ (точки) и $S-C h$ (прямая).

Типичный для данной задачи рисунок либрации, соответствующей требуемому уровню энергии $\widetilde{E}$ в конфигурационном пространстве смешанного импульсно-координатного представления $\left(x_{1}, x_{2}\right)$, приведен на рис. 6 .

Сравнение двух описанных способов (А и Б) вычисления дало полное согласие одного с другим. Для сравнения формул (7) и (16) использовалась логарифмическая шкала, домноженная на $h$ :

$$
J_{0}(h)-h \ln \sqrt{h}=S-C h+O\left(h^{2}\right),
$$

где в качестве $C$ следует взять

$$
C=\ln \left(4 \sqrt{\omega_{1}^{\prime} \omega_{2}^{\prime}}|\dot{X}(0)| \frac{1}{(C n, n)} J^{-1}\right)-\ln \left(\frac{\omega_{1}^{\prime}}{\sqrt{e}}\right) .
$$

Характерный результат сравнения в одной из точек пространства параметров $\left(a, b, \omega_{1}, \omega_{2}\right)$ приведен на рис. 7. 


\section{4. КВАНТОВАЯ ДВОЙНАЯ ЯМА В МАГНИТНОМ ПОЛЕ В СПЕЦИАЛЬНЫХ ТРЕХМЕРНЫХ СЛУЧАЯХ}

Обсудим вопрос, когда соображение, основанное на преобразовании Фурье и приводящее оператор Шредингера в магнитном поле к оператору Шредингера без магнитного поля, может быть реализовано в трехмерном случае.

Рассмотрим потенциал $V\left(x_{1}, x_{2}, x_{3}\right)$ вида

$$
V\left(x_{1}, x_{2}, x_{3}\right)=v_{1}\left(x_{1}\right)+\frac{\omega_{2}^{2} x_{2}^{2}}{2}+\frac{\omega_{3}^{2} x_{3}^{2}}{2}
$$

где $v_{1}\left(x_{1}\right)$ - одномерный потенциал вида двойной ямы.

Мы покажем, что в следующих случаях возможно свести соответствующее спектральное уравнение для оператора Шредингера (2) к спектральной задаче для уравнения Шредингера в потенциале двойной ямы, но в отсутствие магнитного поля:

1) магнитное поле перпендикулярно оси $O x_{1}$;

2) магнитное поле совпадает с направлением оси $O x_{1}$;

3) произвольное направление магнитного поля, но $\omega_{2}=\omega_{3}$.

В случае 1 необходимо использовать следующую калибровку векторного потенциала: $A(x)=\left(0, b_{3} x_{1},-b_{2} x_{1}\right)$ и сделать $h$-преобразование Фурье по переменным $x_{2}$ и $x_{3}$. В случае 2 выбирается $A(x)=\left(0,-b_{1} x_{3}, b_{1} x_{2}\right)$ и осуществляется $h$-преобразование Фурье по переменным $x_{2}$ и $x_{3}$. В случае 3 , используя симметрию потенциала $V$ относительно оси $O x_{1}$, можно считать $b_{3}=0$. Выбрав потенциал в виде $A(x)=\left(b_{2} x_{3}, 0, b_{1} x_{2}\right)$ и сделав частичное $h$-преобразование Фурье по переменной $x_{3}$, получим следующее представление для оператора Шредингера:

$$
\widehat{H}^{\prime}=\left(\hat{p}_{1}-b_{2} \hat{p}_{3}^{\prime}\right)^{2}+\hat{p}_{2}^{2}+\frac{\omega_{3}^{2} \hat{p}_{3}^{\prime}}{2}+v_{1}\left(x_{1}\right)+\frac{\omega_{2}^{2} x_{2}^{2}}{2}+\left(x_{3}^{\prime}-b_{1} x_{2}\right)^{2} .
$$

Подходящей линейной заменой координат $x_{1}, x_{2}, x_{3}^{\prime}$ этот оператор снова принимает “безмагнитный" вид $\widehat{H}=\hat{p}^{2} / 2+\widetilde{V}(x)$.

\section{5. ТУННЕЛИРОВАНИЕ ВОЛНОВЫХ ПАКЕТОВ В КВАНТОВЫХ НАНОПРОВОДАХ}

5.1. Волновые пакеты в одиночном квантовом проводе в однородном магнитном поле. Для моделирования квантового волновода ("одиночного квантового провода") в трехмерном пространстве с координатами $(x, y, z)$, сонаправленного с осью $O x$, имеющего "мягкие стенки" в поперечном сечении и помещенного в постоянное магнитное поле $\mathbf{B}$ с компонентами $\left(b_{x}, b_{y}, 0\right)$, мы используем оператор Шредингера

$$
\widehat{\mathcal{H}}=\frac{1}{2}\left(-i h \frac{\partial}{\partial x}-b_{y} z\right)^{2}-\frac{h^{2}}{2} \frac{\partial^{2}}{\partial y^{2}}+\frac{1}{2}\left(-i h \frac{\partial}{\partial z}-b_{x} y\right)^{2}+U(y, z) .
$$

Здесь $U=\left(\omega^{2} / 2\right)\left(y^{2}+z^{2}\right)$ - потенциал параболического конфайнмента (см. [6]) с частотой $\omega$. Мы выбрали координаты $y, z$ так, чтобы выполнялось условие $b_{z}=0$, и такую калибровку, чтобы потенциал $\mathbf{A}$ магнитного поля $\mathbf{B}$ не зависел от переменной $x: \mathbf{A}=\left(b_{y} z, 0, b_{x} y\right)$. 
Рассмотрим задачу о распространении гауссова пучка в таком волноводе. Соответствующая волновая функция $\Psi(x, y, z, t)$ удовлетворяет уравнению Шредингера

$$
i \hbar \frac{\partial}{\partial t} \Psi=\widehat{\mathcal{H}} \Psi
$$

Сначала приведем известное решение в отсутствие магнитного поля (в случае $\left.b_{x}=b_{y}=b_{z}=0\right)$. Оно легко получается с помощью метода разделения переменных и имеет вид гауссовой "шапочки", бегущей вдоль оси $x$ и расплывающейся вдоль этого направления:

$$
\begin{aligned}
\Psi^{0}(r, t) & =f(t, x) \chi^{0}(y, z), \quad \chi^{0}(y, z)=e^{-\omega\left(y^{2}+z^{2}\right) / 2 h}, \\
f(t, x) & =\frac{A e^{-i E_{0} t / h}}{\sqrt{\alpha+i t}} e^{-(x-P t)^{2} / 2 h(\alpha+i t)} e^{i x P / h} e^{-i P^{2} t / 2 h}, \quad E_{0}=\omega h,
\end{aligned}
$$

где $\alpha$ - положительный параметр, характеризующий ширину “шапочки” в момент времени $t=0$, параметр $A$ - амплитуда.

Решение, обобщающее (19), (20) на случай, когда присутствует магнитное поле $\left(b_{x} \neq 0, b_{z} \neq 0\right)$, имеет вид

$$
\Psi^{0}(x, y, z, t)=\frac{C}{\sqrt{g(t)}} e^{i S(t) / h} e^{i / h\langle P(t), r-R(t)\rangle} e^{i /(2 h)\langle r-R(t), Q(t)(r-R(t))\rangle} .
$$

Здесь $C$ - константа, $r$ - вектор-столбец с компонентами $(x, y, z)$, векторнозначные функции $P(t)=\left(P_{x}(t), P_{y}(t), P_{z}(t)\right), R(t)=(X(t), Y(t), Z(t))$, функции $S(t), g(t)$ и $(3 \times 3)$-матричнозначная функция $Q(t)$ определяются следующим образом:

$$
\begin{gathered}
P_{x}(t)=P=\text { const, } \quad P_{y}(t)=0, \quad P_{z}(t)=0, \\
X(t)=\frac{P \omega^{2}}{\omega_{y}^{2}} t, \quad Y(t)=0, \quad Z(t)=\text { const }=\frac{P b_{y}}{\omega_{y}^{2}}, \\
S(t)=\frac{P^{2} t}{2} \frac{\omega^{2}}{\omega_{y}^{2}}+\frac{h}{2} \beta, \quad g(t)=\frac{b_{y}^{2} \beta}{\omega_{y}^{3}\left(\omega+\omega_{y}\right)}+\mu(t), \quad \mu(t)=\alpha+i t \frac{\omega^{2}}{\omega_{y}^{2}}, \\
Q(t)=\frac{i \frac{b_{x} b_{y}}{\omega_{y} \beta}}{\left(\omega+\omega_{y}\right) g(t)}\left(\begin{array}{ccc}
i \frac{\omega+\omega_{y}}{\beta} & \frac{b_{y}}{\omega_{y}} \\
i \frac{b_{x} b_{y}}{\omega_{y} \beta} & i\left(\frac{b_{y}^{2}\left(\omega_{x}^{2}+\omega \omega_{y}\right)}{\omega_{y}^{3} \beta}+\omega \mu(t)\right) & -\frac{b_{x} \omega_{y}}{\beta} \mu(t) \\
\frac{b_{y}}{\omega_{y}} & i \omega_{y} \mu(t)
\end{array}\right) .
\end{gathered}
$$

Мы обозначили $\omega_{y}^{2}=b_{y}^{2}+\omega^{2}, \omega_{x}^{2}=b_{x}^{2}+\omega^{2}$,

$$
\beta=\sqrt{\frac{\omega_{x}^{2}+\omega_{y}^{2}+\sqrt{\left(\omega_{x}^{2}+\omega_{y}^{2}\right)^{2}-4 \omega^{2} \omega_{y}^{2}}}{2}}+\sqrt{\frac{\omega_{x}^{2}+\omega_{y}^{2}-\sqrt{\left(\omega_{x}^{2}+\omega_{y}^{2}\right)^{2}-4 \omega^{2} \omega_{y}^{2}}}{2}} .
$$


Это решение можно получить разными способами, в частности с помощью теории комплексного ростка Маслова (см. [7]), нам, однако, удобно получить его с помощью частичного преобразования Фурье по переменной $x$. Именно, для функции

$$
\widetilde{\Psi}^{0}\left(p_{x}, y, z, t\right)=\frac{1}{\sqrt{2 \pi h}} \int_{-\infty}^{\infty} \Psi^{0}(x, y, z, t) e^{-i p_{x} x / h} d x
$$

имеем уравнение

$$
\begin{gathered}
i h \frac{\partial \widetilde{\Psi}^{0}}{\partial t}=\widehat{\widetilde{\mathcal{H}}} \widetilde{\Psi}^{0} \\
\widehat{\widetilde{\mathcal{H}}}=-\frac{h^{2}}{2} \frac{\partial^{2}}{\partial y^{2}}+\frac{1}{2}\left(-i h \frac{\partial}{\partial z}-b_{x} y\right)^{2}+\frac{1}{2}\left(p_{x}-b_{y} z\right)^{2}+\frac{\omega^{2}}{2}\left(y^{2}+z^{2}\right)= \\
=-\frac{h^{2}}{2} \frac{\partial^{2}}{\partial y^{2}}+\frac{1}{2}\left(-i h \frac{\partial}{\partial z^{\prime}}-b_{x} y\right)^{2}+\frac{1}{2} \omega^{2} y^{2}+\frac{1}{2} \omega_{y}^{2} z^{\prime 2}+\frac{1}{2} \frac{\omega^{2} p_{x}^{2}}{\omega_{y}^{2}}
\end{gathered}
$$

где

$$
z^{\prime}=z-\frac{b_{y} p_{x}}{\omega_{y}^{2}}
$$

Решение этого уравнения, соответствующее (21), может быть получено с помощью метода разделения переменных и последующего применения известных формул (модели) Дарвина-Фока для гармонического осциллятора в магнитном поле (или с помощью теории комплексного ростка Маслова [7]). Оно имеет вид

$$
\widetilde{\Psi}\left(p_{x}, y, z, t\right)=C e^{-\alpha\left(p_{x}-P\right)^{2} / 2 h} e^{-i \mathcal{E}_{0}\left(p_{x}\right) t / h} \widetilde{\chi}\left(p_{x}, y, z\right),
$$

где

$$
\mathcal{E}_{0}\left(p_{x}\right)=\frac{1}{2} \frac{\omega^{2} p_{x}^{2}}{\omega_{y}^{2}}+\frac{h}{2} \beta, \quad \tilde{\chi}\left(p_{x}, y, z\right)=\exp \left[\frac{i}{h}\left(\frac{1}{2}\left\langle r_{\perp}-R_{\perp}, q\left(r_{\perp}-R_{\perp}\right)\right\rangle\right)\right]
$$

- наименьшее собственное значение и соответствующая ему собственная функция оператора $\widehat{\mathcal{H}}$. В последней формуле

$$
r_{\perp}=\left(\begin{array}{l}
y \\
z
\end{array}\right), \quad R_{\perp}=\left(\begin{array}{c}
0 \\
\frac{p_{x} b_{y}}{\omega_{y}^{2}}
\end{array}\right), \quad q=\frac{1}{\omega+\omega_{y}}\left(\begin{array}{cc}
i \omega \beta & -b_{x} \omega_{y} \\
-b_{x} \omega_{y} & i \omega_{y} \beta
\end{array}\right) .
$$

Заметим, что переменная $p_{x}$ в формуле (26) содержится лишь в (комплексной) фазе, причем зависимость этой фазы от $p_{x}$ квадратичная. Поэтому при нахождении функции $\Psi^{0}$ в переменных $(x, y, z)$ интеграл

$$
\Psi^{0}=\frac{1}{\sqrt{2 \pi h}} \int_{-\infty}^{\infty} \widetilde{\Psi}^{0}\left(p_{x}, y, z, t\right) e^{i p_{x} x / h} d p_{x}
$$

вычисляется точно, что и приводит к формуле (21). Подчеркнем, что представление волнового пакета в виде (26) (и (28)) полезно и с точки зрения задачи о распространении волнового пакета в двух параллельных квантовых проводах. 
При сдвиге координаты $y$ в потенциале $U$ на расстояние $\pm a$, т. е. при рассмотрении задачи с потенциалом $U=\left(\omega^{2} / 2\right)\left((y \pm a)^{2}+z^{2}\right)$, у соответствующих волновых функций изменяется не только аргумент, но и появляется фазовый множитель. Именно, функция $\Psi^{0}(x, y, z, t)$ заменяется на $e^{ \pm i a b_{x} z / h} \Psi^{0}(x, y \pm a, z, t)$, а функция $\widetilde{\Psi}\left(p_{x}, y, z, t\right)-$ на $e^{ \pm i a b_{x} z / h} \widetilde{\Psi}\left(p_{x}, y, z, t\right)$.

\section{2. Волновые пакеты в параллельных квантовых проводах в однород-} ном магнитном поле. Теперь рассмотрим случай, когда имеются два близких параллельных квантовых провода (волновода) и в одном из них распространяется волновой пакет. В этом случае естественно предположить, что потенциал конфайнмента по поперечным переменным $y, z$ имеет вид

$$
V=\frac{1}{8 a^{2}} \omega^{2}\left(y^{2}-a^{2}\right)^{2}+\frac{1}{2} \omega^{2} z^{2} .
$$

Слагаемое $\omega_{1}^{2}\left(y^{2}-a^{2}\right)^{2} / 8$ в потенциале $V$ имеет вид двойной потенциальной ямы в направлении $y$ и задает потенциальный барьер между двумя проводами.

Понятно, что если провода находятся довольно далеко друг от друга, то их можно рассматривать по отдельности, тогда можно использовать осцилляторное приближение и в каждом из проводов пакеты будут вести себя независимо. Потенциал $V$ в окрестности точек $(y=-a, z=0),(y=a, z=0)$ представляется в виде

$$
V=\frac{1}{2} \omega^{2}(y \pm a)^{2}+\frac{1}{2} \omega^{2} z^{2}+O(y \pm a)^{3} .
$$

Ограничимся случаем, когда пакет распространяется в левом проводе.

Сначала рассмотрим случай, когда поле отсутствует. Тогда соответствующая

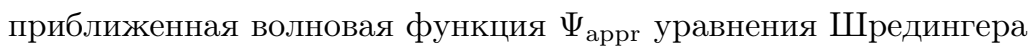

$$
i h \frac{\partial \Psi}{\partial t}=-\frac{h^{2}}{2}\left(\frac{\partial^{2}}{\partial x^{2}}+\frac{\partial^{2}}{\partial y^{2}}+\frac{\partial^{2}}{\partial z^{2}}\right) \Psi+V(y, z) \Psi
$$

имеет вид (19), (20) со сдвинутым аргументом $y \rightarrow y+a: \Psi_{\text {appr }}=f(x, t) \chi^{0}(x, y+a, z)$, $f(x, t)$ определена в (20). Однако на больших временах (в безразмерных переменных порядка $1 / h$ и более) это приближение начинает расходиться с точным ответом и становится существенным, когда происходит туннелирование волнового пакета из одного провода в другой. В силу симметрии потенциала конфайнмента функция $\chi^{0}$ представляется в виде

$$
\chi^{0}(x, y+a, z) \approx \frac{1}{2}\left(\chi^{+}+\chi^{-}\right)
$$

где $\chi^{+}$и $\chi^{-}-$четная и нечетная собственные функции, отвечающие двум минимальным собственным значениям $E^{ \pm}$оператора $\left(h^{2} / 2\right)\left(\partial^{2} / \partial y^{2}+\partial^{2} / \partial z^{2}\right) \Psi+V(y, z)$ :

$$
E^{ \pm}=h \omega+O\left(h^{2}\right), \quad \Delta E=E^{-}-E^{+}=\frac{\omega h}{\sqrt{\pi e}} e^{-\pi J(\omega h / 2) / h}(1+O(h)) .
$$

Таким образом, точное решение задачи Коши для уравнения Шредингера имеет вид

$$
\Psi=\frac{f(t, x)}{2}\left(\chi^{+} e^{-i t E^{+} / h}+\chi^{-} e^{-i t E^{-} / h}\right)=\frac{f(t, x)}{2} e^{-i t E^{+} / h}\left(\chi^{+}+\chi^{-} e^{-i t \Delta E / h}\right) .
$$


В момент времени $t=T \equiv \pi h / \Delta E$ волновая функция $\Psi$ принимает вид

$$
\Psi=f(T) e^{-i T E^{+} / h} \frac{\chi^{+}-\chi^{-}}{2} \approx f(T) e^{-i T E^{+} / h} \chi^{0}(x, y-a, z),
$$

и тем самым волновой пакет, находящийся в “левом" проводе, перемещается в "правый”.

Рассмотрим теперь аналогичный вопрос в случае, когда два параллельных провода помещены в магнитное поле с компонентами $\left(b_{x}, b_{y}, b_{z}\right)$. Мы выберем такую калибровку, в которой векторный потенциал имеет компоненты $\left(b_{y} z-b_{z} y, 0, b_{x} y\right)$. Такую ситуацию удобно сразу рассматривать для уравнения Шредингера в смешанном $\left(p_{x}, y, z\right)$-представлении для функции $\widetilde{\Psi}\left(p_{x}, y, z, t\right)$

$$
\begin{aligned}
i h \frac{\partial \widetilde{\Psi}}{\partial t} & =\widehat{\widetilde{\mathcal{H}}}^{w}, \quad \widehat{\widetilde{\mathcal{H}}}^{w}=\left(-\frac{h^{2}}{2} \frac{\partial^{2}}{\partial y^{2}}+\frac{1}{2}\left(-i h \frac{\partial}{\partial z}-b_{x} y\right)^{2}+W\right) \widetilde{\Psi}, \\
W & =\frac{1}{2}\left(p_{x}-b_{y} z+b_{z} y\right)^{2}+\frac{1}{8 a^{2}} \omega^{2}\left(y^{2}-a^{2}\right)^{2}+\frac{1}{2} \omega^{2} z^{2} .
\end{aligned}
$$

Покажем сначала, что наличие продольной компоненты $b_{z}$ магнитного поля разрушает симметрию "двойной ямы”, более точно, что при $b_{z} \neq 0$ потенциал имеет вид несимметричной двойной или одинарной ямы. Найдем локальные минимумы потенциала $W$ :

$$
\begin{aligned}
& \frac{\partial W}{\partial y}=b_{z}\left(p_{x}-b_{y} z+b_{z} y\right)+\frac{1}{8 a^{2}} \omega^{2}\left(y^{2}-a^{2}\right) y=0, \\
& \frac{\partial W}{\partial z}=-b_{y}\left(p_{x}-b_{y} z+b_{z} y\right)+\omega^{2} z=0 .
\end{aligned}
$$

Выражая $z$ из второго равенства, получаем прямую $z=b_{y}\left(p_{x}+b_{z} y\right) /\left(\omega^{2}+b_{y}^{2}\right)$, проходящую через точки минимума потенциала $W(y, z)$. Заменяя переменную $z$ с помощью этого равенства в потенциале $W(y, z)$, получим значение потенциала на этой прямой

$$
\frac{1}{2} \frac{\omega^{2}}{\omega^{2}+b_{y}^{2}}\left(p_{x}+b_{z} y\right)^{2}+\frac{1}{8 a^{2}} \omega^{2}\left(y^{2}-a^{2}\right)^{2} .
$$

Отсюда ясно, что симметрия потенциала нарушается при $b_{z} \neq 0$. (Исключение составляет точка $p_{x}=0$, которая не играет роли при изучении распространения волнового пакета.) Нарушение симметрии приводит к тому, что носители собственных функций оператора $\widehat{\widetilde{\mathcal{H}}}^{w}$ оказываются локализованными в окрестности либо левой, либо правой ямы, и таким же свойством обладает соответствующий волновой пакет.

Пусть выполнено $b_{z}=0$. Сделаем в уравнении (29) замену (25). Тогда оператор $\widetilde{\widetilde{\mathcal{H}}}^{\text {}}$ примет вид

$$
\widehat{\widetilde{\mathcal{H}}}^{w}=\left(-\frac{h^{2}}{2} \frac{\partial^{2}}{\partial y^{2}}+\frac{1}{2}\left(-i h \frac{\partial}{\partial z^{\prime}}-b_{x} y\right)^{2}+\frac{1}{8 a^{2}} \omega^{2}\left(y^{2}-a^{2}\right)+\frac{1}{2} \omega_{y}^{2} z^{\prime 2}\right)+\frac{1}{2} \frac{\omega^{2} p_{x}^{2}}{\omega_{y}^{2}}
$$

и для построения асимптотики его собственных функций $\chi^{ \pm}\left(p_{x}, y, z^{\prime}\right)$, описывающих два нижних энергетических состояния $\varepsilon^{+}<\varepsilon^{-}$, можно воспользоваться результатами раздела 2. 
Получим, что в смешанном $\left(p_{x}, y, z\right)$-представлении решение $\widetilde{\Psi}$, описывающее распространение волнового пакета, находящегося при $t=0$ в левом проводе, записывается в виде суперпозиции:

$$
\begin{aligned}
& \widetilde{\Psi}\left(p_{x}, y, z, t\right)= \\
& \quad=\frac{C}{2} e^{-\alpha\left(p_{x}-P\right)^{2} / 2 h} e^{-i \omega^{2} p_{x}^{2} t / 2 h \omega_{y}^{2}}\left(\chi^{+}\left(y, z^{\prime}\right) e^{-i \varepsilon^{+} t / h}+\chi^{-}\left(y, z^{\prime}\right) e^{-i \varepsilon^{-} t / h}\right)= \\
& \quad=\frac{C}{2} e^{-i \omega^{2} p_{x}^{2} t / 2 h \omega_{y}^{2}} e^{-i \varepsilon^{+} t / h}\left(\chi^{+}\left(y, z^{\prime}\right)+\chi^{-}\left(y, z^{\prime}\right) e^{-i \Delta \varepsilon t / h}\right), \quad \Delta \varepsilon=\varepsilon^{-}-\varepsilon^{+} .
\end{aligned}
$$

Мы считаем, что функции $\chi^{+}\left(y, z^{\prime}\right) \pm \chi^{-}\left(y, z^{\prime}\right)$ в окрестностях точек $(y, z)=( \pm a, 0)$ приближаются (с учетом сдвига аргумента и фазового сдвига) функциями (27). В обычном $(x, y, z)$-представлении это решение имеет вид

$$
\Psi(x, y, z, t)=\frac{1}{\sqrt{2 \pi h}} \int_{-\infty}^{\infty} \widetilde{\Psi}\left(p_{x}, y, z, t\right) e^{i p_{x} x / h} d p_{x} .
$$

Заметим, что формулы (31) и (32) точные и $\varepsilon^{ \pm}$не зависят от $p_{x}$.

Как и при отсутствии магнитного поля, за время $T=\pi h / \Delta \varepsilon$ во втором слагаемом в (31) накопится фаза $\pi$, что приводит к тому, что волновой пакет из левого провода "перетечет" в правый. Для описания такого перехода можно использовать асимптотические формулы раздела 2. Для $\Delta E$ и $T$ имеем формулы

$$
\Delta \varepsilon=\frac{\omega_{1}^{\prime} h}{\sqrt{\pi e}} e^{-\pi J(\widetilde{E}(h)) / h}(1+O(h)), \quad T=\frac{\pi \sqrt{\pi e}}{\omega_{1}^{\prime}} e^{\pi J(\widetilde{E}(h)) / h}(1+O(h)),
$$

где $\omega_{1}^{\prime}=\sqrt{\omega_{x}^{2}+\omega_{y}^{2}-\sqrt{\left(\omega_{x}^{2}+\omega_{y}^{2}\right)^{2}-4 \omega^{2} \omega_{y}^{2}} / 2}$, а выражение $J(\widetilde{E}(h))$ определяется согласно алгоритму, представленному в разделе 2 . Отсюда находим решение $\Psi$ при $t=0$ и при $t=T$ :

$$
\begin{aligned}
\Psi(x, y, z, 0)= & \frac{1}{\sqrt{2 \pi h}} \int_{-\infty}^{\infty} e^{-\alpha\left(p_{x}-P\right)^{2} / 2 h} \frac{\chi^{+}\left(y, z^{\prime}\right)+\chi^{-}\left(y, z^{\prime}\right)}{2} e^{i p_{x} x / h} d p_{x} \approx \\
\approx & \frac{1}{\sqrt{2 \pi h}} \int_{-\infty}^{\infty} e^{-\alpha\left(p_{x}-P\right)^{2} / 2 h} \widetilde{\chi}\left(y, z-\frac{b_{y} p_{x}}{\omega^{\prime 2}}\right) e^{i p_{x} x / h} d p_{x} \approx \\
\approx & e^{i b_{x} a z / h} \Psi^{0}(x, y+a, z, 0), \\
\Psi(x, y, z, T)= & \frac{e^{-i \varepsilon^{+} T / h}}{\sqrt{2 \pi h}} \int_{-\infty}^{\infty} e^{-\alpha\left(p_{x}-P\right)^{2} / 2 h} e^{-i \omega_{2}^{2} p_{x}^{2} T / 2 h\left(\omega_{2}^{2}+b_{y}^{2}\right)} \times \\
& \quad \times \frac{\chi^{+}\left(y, z^{\prime}\right)-\chi^{-}\left(y, z^{\prime}\right)}{2} e^{i p_{x} x / h} d p_{x} \approx \\
& \frac{e^{-i \varepsilon^{+} T / h}}{\sqrt{2 \pi h}} \int_{-\infty}^{\infty} e^{-\alpha\left(p_{x}-P\right)^{2} / 2 h} e^{-i \omega_{2}^{2} p_{x}^{2} T / 2 h\left(\omega_{2}^{2}+b_{y}^{2}\right)} \times \\
& \times \widetilde{\chi}\left(y, z-\frac{b_{y} p_{x}}{\omega^{\prime 2}}\right) e^{i p_{x} x / h} d p_{x} \approx e^{-i b_{x} a z / h} \Psi^{0}(x, y-a, z, T) .
\end{aligned}
$$


Скажем несколько слов о соотношениях между параметрами задачи. Волновой пакет характеризуется параметром $\alpha$, определяющим его “ширину", и импульсом $P$, характеризующим частоту пространственных осцилляций вдоль оси $x$ и скорость движения пакета по этой оси. Предположим, что длина квантовых проводов равна (в безразмерных единицах) величине $L$. Тогда время пробегания волнового пакета этого расстояния равно $T_{1}=L \omega_{y}^{2} / P \omega^{2}$. За это время амплитуда волнового пакета уменьшится в

$$
\begin{aligned}
& \sqrt{\left(\frac{b_{y}^{2} \beta}{\omega_{y}^{3}\left(\omega+\omega_{y}\right)}+\alpha\right) /\left|\frac{b_{y}^{2} \beta}{\omega_{y}^{3}\left(\omega+\omega_{y}\right)}+\left(\alpha+i T_{1} \frac{\omega^{2}}{\omega_{y}^{2}}\right)\right|}= \\
& =\sqrt{\left(\frac{b_{y}^{2} \beta}{\omega_{y}^{3}\left(\omega+\omega_{y}\right)}+\alpha\right) /\left|\frac{b_{y}^{2} \beta}{\omega_{y}^{3}\left(\omega+\omega_{y}\right)}+\left(\alpha+i \frac{L}{P}\right)\right|}
\end{aligned}
$$

раз. Для того чтобы сказался эффект туннелирования, нужно, чтобы время $T_{1}$ было не меньше, чем (большое) время туннелирования, т. е. $T_{1} \geqslant T$ или

$$
\frac{L \omega_{y}^{2}}{P \omega^{2}} \geqslant \frac{\pi \sqrt{\pi e}}{\omega_{1}^{\prime}} e^{\pi J(\widetilde{E}(h)) / h} \Longleftrightarrow J(\widetilde{E}(h)) \leqslant \frac{h}{\pi} \ln \left(\frac{L \omega_{y}^{2} \omega_{1}^{\prime}}{\pi \sqrt{\pi e} P \omega^{2}}\right) .
$$

При этом, разумеется, пакет не должен сильно "расплыться" по переменной $x$. Более детальный анализ соотношений между параметрами $P, \alpha, L, \omega, b_{x}, b_{y}, a$, проясняющий ситуацию, допускающую одновременно не “сильное расплывание" и туннелирование, мы приведем в последующих публикациях. Сейчас только отметим, что в рассуждениях, связанных с туннелированием, фактически малым параметром является параметр $h \omega$, а для нанопроводов естественно считать $\omega$ большой величиной, и, кроме того, параметры пакета $\alpha$ и $P$ могут принимать различные значения, не связанные с $h, \omega, a, b_{x}, b_{y}$, что и позволяет подобрать параметры задачи соответствующим образом.

Общий вывод, касающийся влияния магнитного поля на эффекты туннелирования волновых пакетов, такой: магнитное поле либо вообще разрушает туннелирование (если $\left.b_{z} \neq 0\right)$, либо, согласно выводам раздела 3 , с ростом компоненты $b_{x}$ (соответствующей переменной $b$ в данном разделе) замедляет, а с ростом $b_{y}$ (растущей вместе с переменной $\omega_{y}^{2}=b_{y}^{2}+\omega^{2}$, соответствующей $\omega_{2}^{2}$ в обозначениях раздела 3 ) ускоряет этот эффект.

\section{6. ЗАКЛЮЧЕНИЕ}

В работе показано, что в двумерном случае задачу о расщеплении нижних уровней для оператора Шредингера с постоянным магнитным полем со специальным потенциалом типа двойной ямы можно свести к подробно изученной спектральной задаче для оператора Шредингера, в котором магнитное поле входит лишь как параметр скалярного потенциала. Методы и формулы, развитые для обычного оператора Шредингера в потенциале двойной ямы, были применены для вычисления экспоненциального расщепления энергетических уровней для исходной задачи и их зависимости от магнитного поля. Полученные формулы применены для анализа эффектов туннелирования волнового пакета в двойном квантовом проводе (сдвоенном квантовом волноводе). 
Благодарности. Авторы благодарны В. Залипаеву за предоставленные результаты прямых расчетов величин расщепления нижних собственных значений, приведенных на рис. 5. Работа поддержана Министерством образования РФ (грант 2.1.1/4540), РФФИ (гранты № 11-01-00973, 11-01-12058) и проектом DFG-RAS (DFG 436 RUS $113 / 572)$.

\section{Список литературы}

[1] В. П. Маслов, Тр. МИАН СССР, 163 (1984), 150-180; A. M. Polyakov, Nucl. Phys. B, 120:3 (1977), 429-458; E. M. Harrell, Commun. Math. Phys., 75:3 (1980), 239-261; B. Helffer, J. Sjöstrand, Commun. Partial Differ. Equations, 9:4 (1984), 337-408; Ann. Inst. H. Poincaré Phys. Théor., 42:2 (1985), 127-212; S. R. Coleman, "The uses of instantons", The Whys of Subnuclear Physics (Erice, Italy, July 23 - August 10, 1977), ed. A. Zichichi, Plenum Press, New York, 1979, 805-916; B. Simon, Ann. Inst. H. Poincaré Sect. A (N.S.), 38:3 (1983), 295-308; Ann. Math. (2), 120:1 (1984), 89-118; S. C. Creagh, J. Phys. A, 27:14 (1994), 4969-4993; С. Ю. Доброхотов, В. Н. Колокольцов, В. П. Маслов, ТМФ, 87:3 (1991), 323-375; S. Yu. Dobrokhotov, V. N. Kolokoltsov, V. P. Maslov, "Quantization of the Bellman equation, exponential asymptotics and tunneling", Idempotent Analysis, Advances in Soviet Mathematics, 13, AMS, Providence, RI, 1992, 1-46.

[2] С. Ю. Доброхотов, В. Н. Колокольцов, ТМФ, 94:3 (1993), 426-434; S. Yu. Dobrokhotov, V. N. Kolokoltsov, J. Math. Phys., 36:3 (1995), 1038-1053.

[3] J. Brüning, S. Yu. Dobrokhotov, E. S. Semenov, Regul. Chaotic Dyn., 11:2 (2006), 167-180.

[4] A. Yu. Anikin, Russ. J. Math. Phys., 20:1 (2013), 1-10; А. А. Аникин, TMФ, 175:2 (2013), 193-204.

[5] В. В. Козлов, ПмМ, 40:3 (1976), 399-407; В. В. Козлов, С. В. Болотин, ПмМ, 42:2 (1978), 245-250.

[6] V.F. Gantmakher, Y.B. Levinson, Carrier Scattering in Metals and Semiconductors, North-Holland, Amsterdam, 1987; Л. И. Магарил, М.В. Энтин, ЖЭТФ, 123:4 (2003), 867-880; М. V. Entin, L. I. Magarill, Phys. Rev. В, 66:20 (2002), 205308, 5 pp.; А. И. Ведерников, А. В. Чаплик, ЖЭТФ, 117:2 (2000), 449-451; В. В. Белов, С. Ю. Доброхотов, Т. Я. Тудоровский, ТМФ, 141:2 (2004), 267-303; В. В. Белов, С. Ю. Доброхотов, В. П. Маслов, Т. Я. Тудоровский, УФН, 175:9 (2005), 1004-1010.

[7] В. П. Маслов, Комплексный метод ВКБ в нелинейных уравнениях, Наука, М., 1977. 